\title{
Electron-positron annihilation freeze-out in the early universe
}

\author{
Luke C. Thomas@, ${ }^{1}$ Ted Dezen, ${ }^{1}$ Evan B. Grohs, ${ }^{2}$ and Chad T. Kishimoto $\oplus^{1,3}$ \\ ${ }^{1}$ Department of Physics and Biophysics, University of San Diego, San Diego, California 92110, USA \\ ${ }^{2}$ Department of Physics, University of California, Berkeley, Berkeley, California 94720, USA \\ ${ }^{3}$ Center for Astrophysics and Space Sciences, University of California, \\ San Diego, La Jolla, California 92093, USA
}

(Received 8 November 2019; accepted 18 February 2020; published 5 March 2020)

\begin{abstract}
Electron-positron annihilation largely occurs in local thermal and chemical equilibrium after the neutrinos fall out of thermal equilibrium and during the big bang nucleosynthesis (BBN) epoch. The effects of this process are evident in BBN yields as well as the relativistic degrees of freedom. We self-consistently calculate the collision integral for electron-positron creation and annihilation using the Klein-Nishina amplitude and appropriate statistical factors for Fermi blocking and Bose enhancement. Our calculations suggest that this annihilation freezes out when the photon-electron-positron-baryon plasma temperature is approximately $16 \mathrm{keV}$, after which its rate drops below the Hubble rate. In the temperature regime near $16 \mathrm{keV}$, we break the assumption of chemical equilibrium between the electrons, positrons, and photons to independently calculate the evolution of the chemical potentials of the electrons and positrons while computing the associated collision integrals at every time step. We find that the electron and positron chemical potentials deviate from the case with chemical equilibrium. While our results do not affect the interpretation of precision cosmological measurements in elucidating the standard cosmological model, these out of equilibrium effects may be important for testing physics beyond the standard model.
\end{abstract}

DOI: 10.1103/PhysRevD.101.063507

\section{INTRODUCTION}

The chronology of the early universe is the story of a hot, dense, and entropy-dominated plasma that may have, at one time, included thermal populations of all particles in the Standard Model (the so-called "primordial soup"). While particle populations are well described by thermal and chemical equilibria, the standard cosmological model incorporates phase transitions that change the nature of the fundamental forces in the Standard Model as well as epochs where various processes freeze-out of equilibrium. Freeze-out from equilibrium is not an instantaneous process, resulting in deviations of particle phase-space distributions as the approximations of local thermal and chemical equilibrium become invalid [1-4].

Upcoming high-precision cosmological observations $[5,6]$ introduce the promise of the early universe as a testing ground for the Standard Model and the standard cosmological model. Out-of-equilibrium effects that occur when freeze-out is not instantaneous are an especially

Published by the American Physical Society under the terms of the Creative Commons Attribution 4.0 International license. Further distribution of this work must maintain attribution to the author(s) and the published article's title, journal citation, and DOI. Funded by SCOAP. promising regime for revealing beyond Standard Model (BSM) physics [7-17].

The well-studied epochs of weak decoupling and weak freeze-out occur when the temperature of the photonelectron-positron-baryon plasma, $T$, is approximately an $\mathrm{MeV}$ [18]. Weak decoupling ( $T \sim 3 \mathrm{MeV}$ ) occurs when neutrinos no longer efficiently exchange energy with the plasma so they are no longer in thermal equilibrium. Weak freeze-out $(T \sim 0.7 \mathrm{MeV})$ occurs when the charged current lepton-nucleon processes inefficiently interconvert protons to neutrons, freezing-out the neutron-to-proton ratio as they fall out of chemical equilibrium with the leptons. These processes occur over many Hubble times (expansion timescales), requiring a self-consistent treatment of the Boltzmann evolution of neutrino distribution functions and a nuclear network to describe the consequent big bang nucleosynthesis (BBN) [1]. The results of these computationally intensive calculations may be observable in the energy density of neutrinos (as parametrized by $N_{\text {eff }}$ ) and in primordial abundances as the next generation of cosmic microwave background (CMB) observatories and thirtymeter telescopes come on-line $[5,6]$.

Careful analysis of this epoch reveals a variety of Standard Model processes that affect the value of $N_{\text {eff }}$ and BBN yields because weak decoupling and weak freeze-out are not instantaneous. The better known of 
these effects is the increase of $N_{\text {eff }}$ from its standard, instantaneous decoupling value of 3 to the accepted value of 3.046 due to a variety of effects including electronpositron annihilation, out-of-equilibrium neutrino upscattering off the photon-electron-positron-baryon plasma, and finite temperature QED radiative corrections to the inmedium electron and photon masses [1,19-22]. Other work has looked into the effects of neutrino quantum kinetic behavior [2,3] and nonstandard cosmological models including the introduction of nonzero lepton numbers [23]. BBN yields are also affected through their influence on neutrino distribution functions and the time-temperature relationship throughout the BBN epoch.

These in-depth studies are necessary to disentangle out-of-equilibrium Standard Model effects from BSM physics, both of which may have an observable signal in $N_{\text {eff }}$ and the BBN yields. During the BBN epoch, electron-positron creation becomes kinematically suppressed compared to annihilation, driving the equilibrium process $-e^{-} e^{+} \rightleftharpoons \gamma \gamma$ - to the right, resulting in the number densities of electrons and positrons plummeting. In the standard cosmological model, BBN yields and $N_{\text {eff }}$ are calculated as if this process always occurs in local thermal and chemical equilibrium [24].

The goal of this work is to closely examine the process of electron-positron annihilation as it freezes out from its local chemical equilibrium. We will determine if and when the electron and positron number densities freeze-out, investigate the consequent effects on cosmological observables, and speculate on whether the effects of freeze-out should affect the standard BBN calculations. To do so, we calculate the collision integral for electron-positron annihilation using the Klein-Nishina amplitude with the appropriate Fermi-blocking/Bose-enhancing statistical factors. Appendix B details our methodology to self-consistently and efficiently calculate these integrals using methods similar to those in Ref. [1].

Throughout this work we use the convention that $\hbar=c=k_{B}=1$. In Sec. II, we use physical principles, including chemical equilibrium between the electrons and positrons, to introduce the standard cosmological computation to evolve the electron and positron distributions. Next, in Sec. III, we calculate the collision integral associated with electron-positron annihilation to infer when freeze-out occurs. We subsequently use the collision integral to separately evolve the electron and positron distributions without the assumption that the charged leptons maintain chemical equilibrium in Sec. IV. In Sec. V we will discuss the results and present conclusions. Appendix A details the equations of motion derived and solved in this work.

\section{CHEMICAL EQUILIBRIUM}

As evidenced by the cosmic microwave background, the primordial plasma is well described by local thermodynamic equilibrium. Scattering between photons, electrons, positrons, and baryons remains fast compared to the Hubble rate, maintaining thermal distributions well into the BBN epoch. This means the photons, electrons, positrons, and baryons share a single plasma temperature $T$, and the electron/positron distributions are Fermi-Dirac. When further assuming the process $e^{-} e^{+} \rightleftharpoons \gamma \gamma$ to be in local chemical equilibrium, the chemical potentials of the involved particles maintain $\mu_{e^{-}}+\mu_{e^{-}}-2 \mu_{\gamma}=0$, stipulating $\mu_{e^{+}}=-\mu_{e^{-}}$. For chemical potentials $\mu_{e^{ \pm}}$, we define the electron and positron degeneracy parameters $\eta^{-} \equiv \mu_{e^{-}} / T$ and $\eta^{+} \equiv \mu_{e^{+}} / T$. Chemical equilibrium is implemented by setting $\eta^{-}=\eta$ and $\eta^{+}=-\eta$.

Given local thermodynamic and chemical equilibrium, the electron and positron momentum/energy distributions are

$$
\begin{aligned}
& f_{e^{-}}(p)=\frac{1}{e^{E_{p} / T-\eta}+1}, \\
& f_{e^{+}}(p)=\frac{1}{e^{E_{p} / T+\eta}+1},
\end{aligned}
$$

where $E_{p}=\sqrt{p^{2}+m_{e}^{2}}$. The evolution of the electron and positron distributions is encapsulated in the evolution of $T$ and $\eta$. To evolve these quantities, we introduce two physical principles-conservation of comoving entropy, and the effects of weak interactions on the relative number of electrons and positrons.

The early universe is homogeneous and isotropic, precluding spatial heat flows. In the absence of out-ofequilibrium decays, the total entropy in a comoving volume is conserved,

$$
\frac{d}{d t}\left[s a^{3}\right]=0 .
$$

Here $a$ is the scale factor in the Friedmann-LemaitreRobertson-Walker metric and entropy density is

$$
s=\frac{\rho_{\mathrm{th}}+P_{\mathrm{th}}}{T}-\eta n_{e^{-}}+\eta n_{e^{+}} .
$$

The electron and positron number densities are denoted $n_{e^{ \pm}}$, and the quantities $\rho_{\text {th }}$ and $P_{\text {th }}$ are energy density and pressure of the thermally coupled particles in the photonelectron-positron-baryon plasma. We treat the neutrinos and antineutrinos as being thermally decoupled from the plasma. While the neutrino seas remain largely thermally coupled to the plasma for $T \gtrsim 1 \mathrm{MeV}$, there is subpercent entropy transfer between the neutrinos and the plasma and orders of magnitude less entropy generation from out-ofequilibrium scattering between neutrinos and the plasma for $T \lesssim 1 \mathrm{MeV}$ [1]. These effects are important in fully understanding the evolution of the universe around the weak decoupling and BBN epochs [1-4], but are computationally expensive and beyond the scope of this work. 
The scale factor evolves according to the Friedmann equation,

$$
\frac{1}{a} \frac{d a}{d t}=\sqrt{\frac{8 \pi}{3 m_{\mathrm{pl}}^{2}} \rho}
$$

where $m_{\mathrm{pl}}$ is the Planck mass. The total energy density of the universe, $\rho$, includes the contents of the plasma along with the neutrinos, dark matter, and dark energy, though the latter two are a negligible fraction of the total energy density at this epoch.

The number densities of electrons and positrons are affected by the expansion of the universe, creation and annihilation, and charged-current weak interactions. Creation and annihilation rates are fast compared to the weak rates, but are computationally expensive to calculate. To avoid the need to self-consistently calculate these rates with the evolution, we introduce the quantity $\left(n_{e^{-}}-n_{e^{+}}\right) a^{3}$, which is insensitive to both the expansion of the universe and electromagnetic processes. Electromagnetic processes preserve the relative number of electrons and positrons, so the evolution of this quantity does not depend on the collision integral, even when the assumption of chemical equilibrium is broken.

Weak interactions that interconvert neutrons and protons are the only processes that cause $\left(n_{e^{-}}-n_{e^{+}}\right) a^{3}$ to change. During the BBN epoch, these processes are

$$
\begin{aligned}
& \nu_{e}+n \rightleftharpoons p+e^{-}, \\
& e^{+}+n \rightleftharpoons p+\bar{\nu}_{e}, \\
& n \rightleftharpoons p+\bar{\nu}_{e}+e^{-} .
\end{aligned}
$$

When the above reactions proceed to the right, $\left(n_{e^{-}}-\right.$ $\left.n_{e^{+}}\right) a^{3}$ increases, while the reverse reactions cause it to decrease. The rate of change of this quantity is

$$
\begin{aligned}
\frac{d}{d t}\left[\left(n_{e^{-}}-n_{e^{+}}\right) a^{3}\right]= & n_{n} a^{3}\left(\lambda_{\nu_{e} n}+\lambda_{e^{+} n}+\lambda_{n}\right) \\
& -n_{p} a^{3}\left(\lambda_{e^{-} p}+\lambda_{\bar{\nu}_{e} p}+\lambda_{\bar{\nu}_{e} e^{-} p}\right) .
\end{aligned}
$$

The rates $\lambda_{i}$, with $i$ indicating the reactants, are computed in Ref. [25]. Rather than directly computing the number densities of neutrons $\left(n_{n}\right)$ and protons $\left(n_{p}\right)$, we can recast Eq. (9) in terms of the electron fraction

$$
Y_{e}=\frac{n_{p}}{n_{b}}=\frac{n_{e^{-}}-n_{e^{+}}}{n_{b}}
$$

resulting in

$$
\begin{aligned}
\frac{1}{n_{b} a^{3}} \frac{d}{d t}\left[\left(n_{e^{-}}-n_{e^{+}}\right) a^{3}\right]= & \left(1-Y_{e}\right)\left(\lambda_{\nu_{e} n}+\lambda_{e^{+} n}+\lambda_{n}\right) \\
& -Y_{e}\left(\lambda_{e^{-} p}+\lambda_{\bar{\nu}_{e} p}+\lambda_{\bar{\nu}_{e} e^{-} p}\right),
\end{aligned}
$$

where $n_{b}$ is the number density of baryons and the quantity $n_{b} a^{3}$ is constant. The right-hand side comes from the rate of change of $Y_{e}$ [25],

$$
\begin{aligned}
\frac{d Y_{e}}{d t}= & \left(1-Y_{e}\right)\left(\lambda_{\nu_{e} n}+\lambda_{e^{+} n}+\lambda_{n}\right) \\
& -Y_{e}\left(\lambda_{e^{-} p}+\lambda_{\bar{\nu}_{e} p}+\lambda_{\bar{\nu}_{e} e^{-} p}\right)
\end{aligned}
$$

which accounts for the production and destruction of electrons and positrons by the weak interactions.

Together, the conservation of comoving entropy [Eq. (3)], the evolution of the difference in comoving numbers of electrons and positrons due to weak interactions [Eq. (11)], the evolution of $Y_{e}$ [Eq. (12)], and the Friedmann equation [Eq. (5)] form a system of coupled differential equations for dependent variables $T, \eta, Y_{e}$, and $a$ (see Sec. I of Appendix A). It should be noted that the definition of $Y_{e}$, Eq. (10), is an integral relationship between all four dependent variables, so while the four aforementioned differential equations are not independent, we find it more efficient to simultaneously use them to evolve the four variables.

We elect an initial plasma temperature $T_{i}=10 \mathrm{MeV}$, sufficiently high that we are assured that the electronpositron annihilation rate is much greater than the Hubble rate. At this temperature, the weak interactions [Eqs. (6)-(8)] are in chemical equilibrium so that the initial electron fraction is [18]

$$
Y_{e, i}=\left[\exp \left(-\frac{m_{n}-m_{p}}{T_{i}}+\eta_{i}\right)+1\right]^{-1}
$$

The initial degeneracy parameter, $\eta_{i}$, is chosen to be consistent with the observed baryon-to-photon ratio from CMB observations [26],

$$
\frac{n_{b, f}}{n_{\gamma, f}}=\frac{n_{b, f}}{\frac{2 \zeta(3)}{\pi^{2}} T_{f}^{3}}=6.1 \times 10^{-10},
$$

where $\zeta(3) \approx 1.20206$ and the subscript $f$ indicates the values at the end of the calculation. (Here, we assume there is no BSM physics that changes the baryon-to-photon rates between the $\mathrm{keV}$ scale and recombination [7].) Since $n_{b} a^{3}$ is conserved, the initial baryon number density is

$$
n_{b_{i}}=\left(6.1 \times 10^{-10}\right) \frac{2 \zeta(3)}{\pi^{2}}\left(\frac{a_{f} T_{f}}{a_{i} T_{i}}\right)^{3} T_{i}^{3} .
$$

In the standard cosmological model $[18,27]$, 


$$
T_{f} a_{f}=\left(\frac{11}{4}\right)^{\frac{1}{3}} T_{i} a_{i}
$$

which accounts for the heating of the plasma when the rest mass energy in electrons and positrons is liberated by annihilation. The initial baryon number can be related to the initial degeneracy parameter through integration of the Fermi-Dirac distribution,

$$
n_{b, i}=\frac{1}{Y_{e, i}}\left(n_{e^{-}}-n_{e^{+}}\right)_{i} \approx \frac{1}{Y_{e, i}} \frac{1}{3} \eta_{i} T_{i}^{3},
$$

assuming that $T \gg m_{e}$ and $\eta_{i} \ll 1$. Equations (13)-(17) produce the initial conditions to evolve $T, \eta, Y_{e}$, and $a$ through the epoch where annihilation is important.

Figure 1 presents the results of this chemical equilibrium solution. The number of particles in a comoving volume for each species in the photon-electron-positron-baryon plasma is plotted as a function of decreasing plasma temperature. When the plasma temperature drops below about an $\mathrm{MeV}$, pair production becomes energetically suppressed, causing the numbers of electrons and positrons to fall precipitously, with positrons tracking electrons until electron numbers abruptly level off and positron numbers continue to plummet. As seen in Fig. 1, this difference is driven by rapid growth in $\eta$.

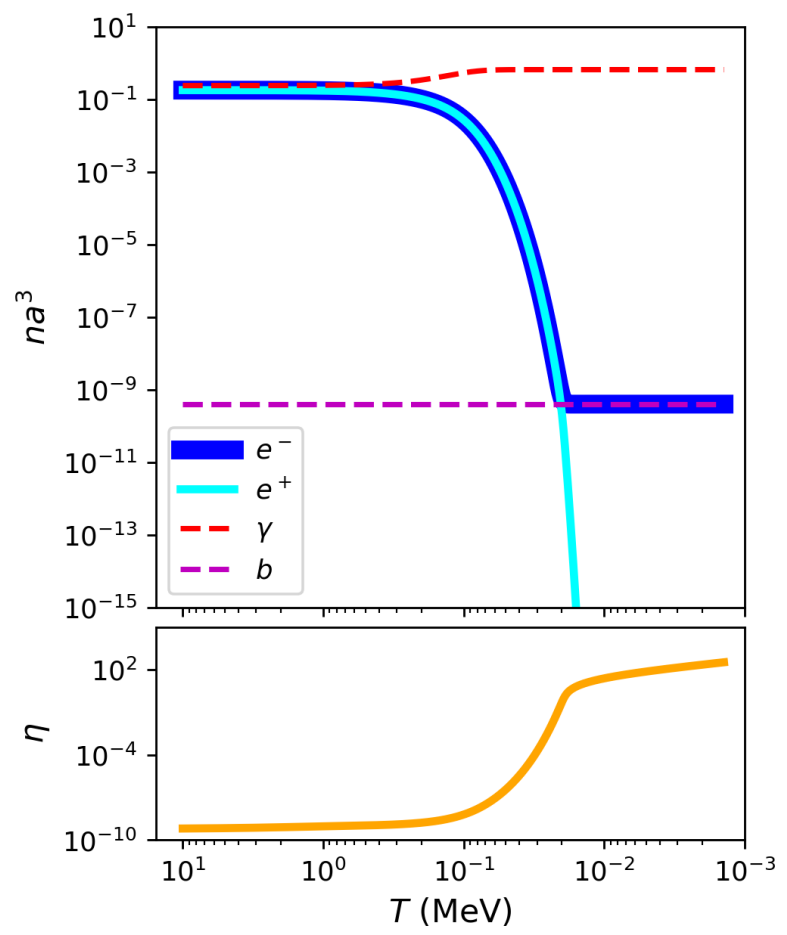

FIG. 1. Top panel: number of particles in a comoving volume for electrons (thick solid curve), positrons (thin solid curve), photons (upper dashed curve), and baryons (lower dashed curve) as a function of plasma temperature. Bottom panel: electron degeneracy parameter, $\eta$ (with $\eta^{-}=\eta$ and $\eta^{+}=-\eta$ ).
Local chemical equilibrium should hold so long as the creation/annihilation rates for $e^{-} e^{+} \rightleftharpoons \gamma \gamma$ remain fast relative to the Hubble rate. The rapid decrease in electron and positron numbers causes the equilibrium creation/ annihilation rate to similarly fall off. The following section utilizes the equilibrium solution to calculate the annihilation rate to assess the possibility of annihilation freeze-out.

One minor issue with this calculation is the lack of a BBN nuclear network calculation. BBN would "protect" neutrons from undergoing free neutron decay by locking up the free neutrons primarily in ${ }^{4} \mathrm{He}$ nuclei. In the standard cosmological model, this occurs at $T \sim 100 \mathrm{keV}$ and results in $Y_{e} \approx 0.88$ at late times (while in our calculation $Y_{e} \rightarrow 1$ as free neutron decay eventually converts every neutron into a proton). Modern BBN codes [28-30] assume that the electrons and positrons remain in thermal equilibrium with the plasma and are in chemical equilibrium with each other. As we do here, they relate the evolution of the electron/positron chemical potential to charge conservation (the net number of electrons over positrons is equal to the number of protons), but in addition to the weak interaction, they also include nuclear reactions.

To ensure that this approximation has negligible effects on our results, we performed the same calculation with a constant $Y_{e}$ equal to its initial value throughout the evolution and found no significant changes in the conclusions. Having bracketed the solution that includes BBN, we expect that self-consistently following the nuclei through BBN will not affect the conclusions of this work.

\section{ANNIHILATION RATES}

To determine when the annihilation process $e^{-} e^{+} \rightarrow \gamma \gamma$ freezes out, we compute the fractional rate of change of the number density of electrons and positrons, due solely to annihilation,

$$
\mathcal{R}_{e^{ \pm}} \equiv \frac{1}{n_{e^{ \pm}}}\left|\frac{d n_{e^{ \pm}}}{d t}\right|_{e^{-} e^{+} \rightarrow \gamma \gamma}
$$

The time derivative of the number densities can be calculated directly from the collision integral,

$$
\begin{aligned}
\left.\frac{d n_{e^{ \pm}}}{d t}\right|_{e^{-} e^{+} \rightarrow \gamma \gamma} & =\frac{d}{d t} \int_{0}^{\infty} \frac{p^{2} d p}{2 \pi^{2}} f_{e^{ \pm}}(p) \\
& =-\int_{0}^{\infty} \frac{p^{2} d p}{2 \pi^{2}} C_{a}(p) .
\end{aligned}
$$

Here, $C_{a}$ is the collision integral for the annihilation pathway,

$$
\left.\frac{d f_{e^{ \pm}}}{d t}\right|_{e^{-} e^{+} \rightarrow \gamma \gamma}=-C_{a}(p)
$$




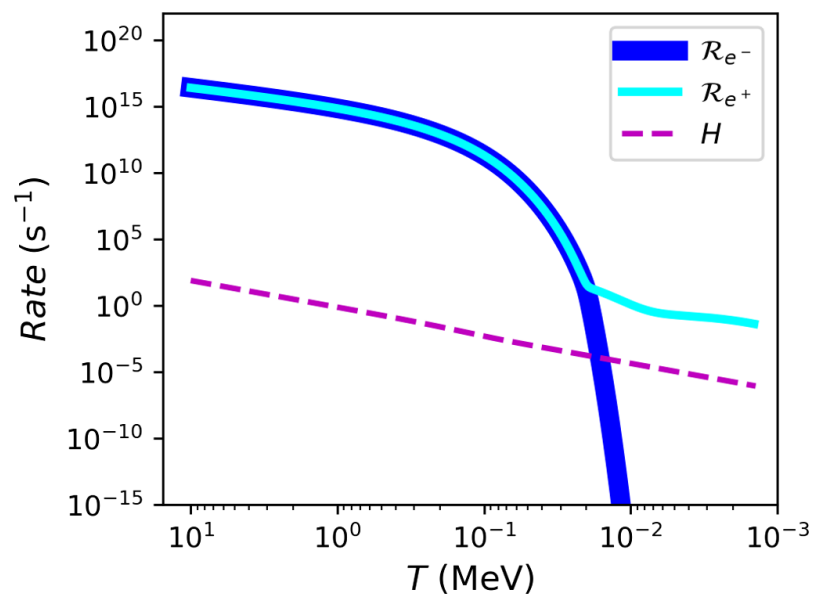

FIG. 2. Annihilation rates for electrons $\left(\mathcal{R}_{e^{-}}\right.$, thick solid line) and positrons ( $\mathcal{R}_{e^{+}}$, thin solid line) as a function of decreasing plasma temperature. Also plotted is the Hubble rate (dashed line) for comparison. Each of these rates are calculated using the results from the chemical equilibrium solution.

which is one of many terms in the Boltzmann equation for the rate of change of $f_{e^{ \pm}}$. Appendix B outlines the computation of the collision integral.

Figure 2 presents $\mathcal{R}_{e^{ \pm}}$calculated using $T, \eta, Y_{e}$, and $a$ from the chemical equilibrium solution. Because of the paucity of positrons, $\mathcal{R}_{e^{-}}$drops below the Hubble rate at $16 \mathrm{keV}$, so at this time the universe expands much faster than the rate at which electrons annihilate with positrons. However, $\mathcal{R}_{e^{+}}$remains many orders of magnitude faster than the Hubble rate as it is much more likely for a positron to annihilate on the significantly more abundant electrons, so essentially every positron will annihilate with an electron.

\section{OUT OF EQUILIBRIUM}

With annihilation freeze-out estimated to occur at $16 \mathrm{keV}$, we lift the assumption of local chemical equilibrium and reexamine this period more closely. Electrons and positrons continue to efficiently scatter with the plasma, so their distributions are still Fermi-Dirac,

$$
f_{e^{ \pm}}(p)=\frac{1}{e^{E_{p} / T-\eta^{ \pm}}+1},
$$

but allowing for deviations from chemical equilibrium implies that the electron and positron degeneracy parameters evolve separately. With independent electron and positron degeneracy parameters, we need to reevaluate the four coupled equations of motion used in the chemical equilibrium solution [Eqs. (3), (5), (11), (12)] to account for the separate degeneracy parameters (see Sec. II in Appendix A). In addition, we introduce the time evolution of the sum of electron and positron numbers in a comoving volume, $\left(n_{e^{-}}+n_{e^{+}}\right) a^{3}$, which is modified by weak interactions as well as creation and annihilation processes:

$$
\begin{aligned}
\frac{d}{d t}\left[\left(n_{e^{-}}+n_{e^{+}}\right) a^{3}\right]= & n_{n} a^{3}\left(\lambda_{\nu_{e} n}-\lambda_{e^{+} n}+\lambda_{n}\right) \\
& +n_{p} a^{3}\left(-\lambda_{e^{-} p}+\lambda_{\bar{\nu}_{e} p}-\lambda_{\bar{\nu}_{e} e^{-} p}\right) \\
& +2 a^{3} \mathcal{N} .
\end{aligned}
$$

The first two terms on the right-hand side account for the creation and absorption of electrons and positrons in the weak interactions, and $\mathcal{N}$ accounts for out-of-equilibrium effects from creation/annihilation,

$$
\mathcal{N}=\left.\frac{d n_{e^{-}}}{d t}\right|_{e^{-} e^{+} \rightleftharpoons \gamma \gamma}=\left.\frac{d n_{e^{+}}}{d t}\right|_{e^{-} e^{+} \rightleftharpoons \gamma \gamma} .
$$

To determine $\mathcal{N}$, we self-consistently calculate the collision integral for creation/annihilation, $C(p)$,

$$
\left.\frac{d f_{e^{ \pm}}}{d t}\right|_{e^{-} e^{+} \rightleftharpoons \gamma \gamma}=C_{c}(p)-C_{a}(p) \equiv C(p),
$$

where $C_{a}(p)$ is the collision integral for the annihilation pathway as in the previous section, and $C_{c}(p)$ is for the creation pathway. It follows that

$$
\mathcal{N}=\int_{0}^{\infty} \frac{p^{2} d p}{2 \pi^{2}} C(p) .
$$

Further details on the collision integral calculations can be found in Appendix B.

Together, Eqs. (3), (5), (11), (12), and (22) yield a system of coupled differential equations for dependent variables $T$, $\eta^{-}, \eta^{+}, Y_{e}$, and $a$. As with the chemical equilibrium solution, the definition of $Y_{e}$, Eq. (10), is an integral relationship between all the dependent variables. While this statement again means that the differential equations are not independent, we simultaneously solve the five differential equations for the five variables, as we did in the chemical equilibrium solution.

Figure 2 shows that the creation and annihilation rates are many orders of magnitude greater than the Hubble rate for $T>16 \mathrm{keV}$, which would require far too many time steps to integrate these equations from $T=10 \mathrm{MeV}$ as we did with the chemical equilibrium solution. However, because we expect chemical equilibrium to hold for $T>16 \mathrm{keV}$, we can use the chemical equilibrium solution to determine initial conditions for this nonequilibrium solution.

Figure 3 presents the solution to these fully nonequilibrium conditions between 24 and $12 \mathrm{keV}$, lasting about a Hubble time and bracketing the period of interest. The initial conditions for $Y_{e}$ and $a$ are taken from the chemical equilibrium solution at $T=24 \mathrm{keV}$, and the initial 


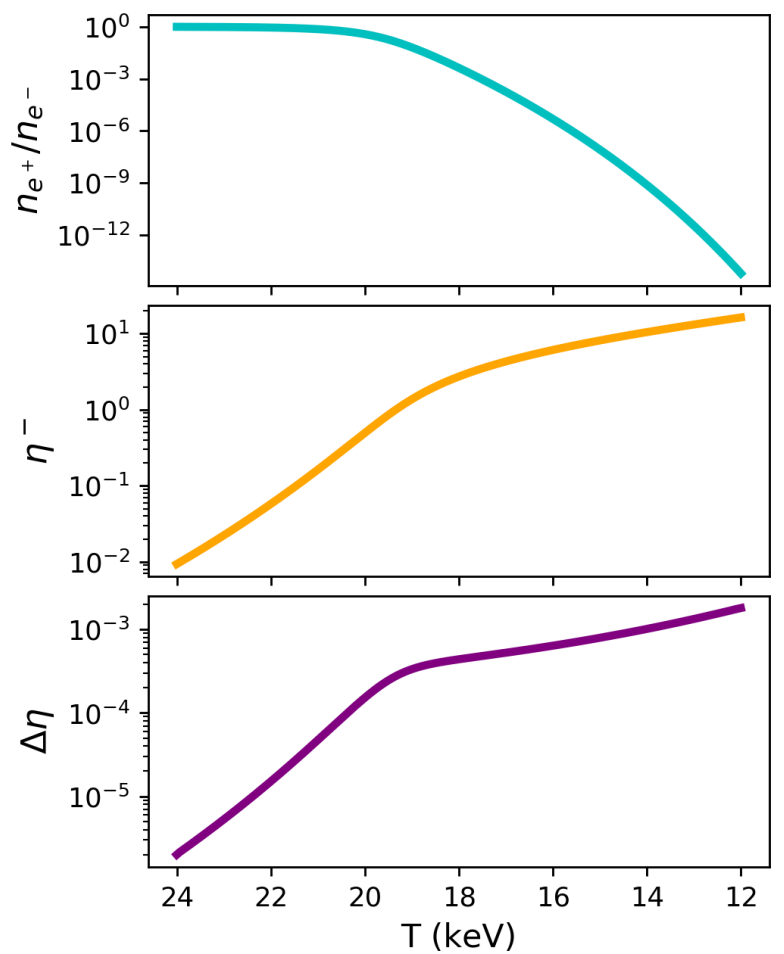

FIG. 3. Results from the nonequilibrium solution using the chemical equilibrium solution as the initial condition at $T=24 \mathrm{keV}$. From top to bottom, the plots show the positronto-electron ratio $\left(n_{e^{+}} / n_{e^{-}}\right)$, electron degeneracy parameter $\left(\eta^{-}\right)$, and the deviation of the electron and positron degeneracy parameters from equilibrium, $\Delta \eta=\eta^{-}+\eta^{+}$, for plasma temperatures from $24 \mathrm{keV}$ to $12 \mathrm{keV}$.

degeneracy parameters for electrons and positrons are derived from $\eta$ in the chemical equilibrium solution at $T=24 \mathrm{keV}$ with $\eta^{-}=\eta$ and $\eta^{+}=-\eta$.

Deviation from $\eta^{+}=-\eta^{-}$indicates a shift away from local chemical equilibrium, so the quantity $\Delta \eta=\eta^{-}+\eta^{+}$ parametrizes the magnitude of any such out-of-equilibrium effect. The initial conditions set the initial value of $\Delta \eta=0$, but the nonequilibrium solution immediately deviates from this initial condition. Throughout the evolution, $\Delta \eta \ll \eta^{-}$, and the electron degeneracy parameter in the nonequilibrium solution $\left(\eta^{-}\right)$differs from the equilibrium solution ( $\eta$ in Fig. 1) by an amount on the order of $\Delta \eta$. As a result, the positron-to-electron ratio and the electron chemical potential as shown in Fig. 3 slightly differ from the corresponding values in the chemical equilibrium solution with a fractional difference on the order of $\Delta \eta$.

To test the validity of taking the initial conditions directly from the chemical equilibrium solution, we repeated the process of determining initial conditions at $25 \mathrm{keV}$, and then evolved the nonequilibrium equations. Once again, the initial conditions are chosen such that the initial $\Delta \eta=0$ and the nonequilibrium solution immediately deviates from this value. We found that this solution was consistent with the results presented in Fig. 3 for $24 \mathrm{keV}<T<12 \mathrm{keV}$.
There are two possible observable consequences of this out-of-equilibrium process: BBN yields and $N_{\text {eff }}$. The light element abundances formed in $\mathrm{BBN}$ are sensitive to the time-temperature relationship and the neutron-to-proton ratio during the $\mathrm{BBN}$ epoch $(\sim 100 \mathrm{keV})$, both of which are influenced by the electron and positron distribution functions. The observationally inferred value of $N_{\text {eff }}$ is affected by the electron/positron number densities and outof-equilibrium entropy transfer between the neutrinos and the plasma. We find that electron-positron annihilation freeze-out occurs at a sufficiently late time that there should be negligible effects on BBN yields and $N_{\text {eff }}$.

Out-of-equilibrium effects are small $\left(\Delta \eta \sim 10^{-6}\right)$ at $T \approx 24 \mathrm{keV}$, yet the BBN yields are set before this time, at higher plasma temperatures when $\Delta \eta$ is many orders of magnitude smaller. (See, e.g., Refs. [1,31] for a discussion of BBN with out-of-equilibrium Boltzmann neutrino transport.) As a result, we expect the BBN yields calculated with the standard assumption of thermal and chemical equilibrium in the electron/positron distributions to be insensitive to the loss of local chemical equilibrium observed in this calculation.

$N_{\text {eff }}$ parametrizes the energy density in ultrarelativistic particles (photons and neutrinos/antineutrinos in the standard cosmological model), $\rho_{\text {rad, }}$, at the epoch of photon decoupling,

$$
\rho_{\mathrm{rad}}=\left[2+\frac{7}{4}\left(\frac{4}{11}\right)^{4 / 3} N_{\mathrm{eff}}\right] \frac{\pi^{2}}{30} T^{4} .
$$

The value of $N_{\text {eff }}=3$ is predicated on a number of simplifying assumptions [27] that neglect a number of effects. Out-of-equilibrium scattering between neutrinos and electron/positrons distorts neutrino spectra, transferring entropy from the plasma to the neutrinos, and increases the total entropy of the universe [1]. Nonzero electron/positron mass and finite-temperature QED affect the equation of state for electrons, positrons, and photons in the plasma. Together, these effects increase $N_{\text {eff }}$ by $\Delta N_{\text {eff }} \equiv N_{\text {eff }}-3 \approx$ $0.046[1-3,19]$. The initial nonzero electron/positron degeneracy parameters are responsible for a negligible increase, $\Delta N_{\text {eff }} \approx 10^{-19}$, due to a higher initial plasma entropy. On the other hand, the nonzero final number density of electrons (and negligible number density of positrons) results in a negligible decrease, $\Delta N_{\text {eff }} \approx-10^{-7}$, because some of the entropy remains in the electron seas at recombination.

\section{DISCUSSION AND CONCLUSIONS}

In this work, we found that the electrons and positrons remain in local chemical equilibrium throughout the BBN epoch and that this chemical equilibrium persists until the plasma temperature is approximately $16 \mathrm{keV}$, when the electron annihilation rate drops below the Hubble rate. This annihilation freeze-out occurs after the BBN yields have 
been set and when the number densities of electrons and positrons are sufficiently low such that the value of $N_{\text {eff }}$ is unaffected. To reach this conclusion, we self-consistently calculated the collision integral for electron-positron annihilation using the Klein-Nishina amplitude and the appropriate Fermi-blocking and Bose-enhancing statistical factors to take into account out-of-equilibrium effects.

The currently accepted value of $N_{\text {eff }}$ is predicated on the complete annihilation of the electrons and positrons from thermal number densities that are nearly equal to the photon number density to essentially zero [27]. The slight changes in the degeneracy parameters of electrons and positrons when the chemical equilibrium approximation is abandoned does not change this conclusion-so as long as the standard cosmological model is assumed, annihilation freeze-out should have a negligible effect on the value of $N_{\text {eff }}$.

BBN calculations assume chemical equilibrium between the electrons and positrons with the photons to model the creation/annihilation process $\left(e^{-} e^{+} \rightleftharpoons \gamma \gamma\right)$ [24]. We found that this approximation holds well throughout the BBN epoch, so BBN calculations in the standard cosmological model are not affected by the specifics of the annihilation freeze-out process.

BSM physics or nonstandard cosmological models may push the annihilation freeze-out earlier (or nuclear freezeout earlier) so that they may influence these cosmological observables. Two possibilities that would require BSM physics to alter the standard cosmological model are a higher baryon-to-photon ratio during the $\mathrm{BBN}$ epoch and a higher effective electron mass.

While the baryon-to-photon ratio is directly measured from the CMB, this value is indicative of the baryon-tophoton ratio in the epochs immediately preceding photon decoupling, not necessarily at earlier epochs [7]. Although the deuterium and helium BBN yields and observations appear to form a concordance between the baryon-tophoton ratio at recombination and the $\mathrm{BBN}$ epochs, the lithium problem may suggest a more complicated evolution of this quantity.

For example, the out-of-equilibrium decay of a BSM particle during or after the BBN epoch could generate entropy in the plasma, significantly decreasing the baryonto-photon ratio [12]. In this scenario, there would be a higher baryon-to-photon ratio during the BBN epoch, leading to a higher annihilation freeze-out temperature. This should result in larger number densities of electrons and positrons throughout the BBN epoch when compared to the standard cosmological model. Solely due to this effect, we would expect to see a decrease in $N_{\text {eff }}$ because some entropy will remain in the electron and positron seas. In addition, an increased positron number density throughout the BBN epoch would reduce the number density of neutrons through positron capture, $e^{+}+n \rightarrow p+\bar{\nu}_{e}$, which has no energy threshold [27]. This more pronounced scarcity of neutrons will suppress the production of the light elements in BBN. These considerations would need to be considered alongside the effects that involve the specifics of the BSM particle decay-decay pathways into high-energy out-of-equilibrium neutrinos and the late entropy generation that dilutes thermal neutrino populations - which also influence the inferred value of $N_{\text {eff }}$ and BBN yields.

Nonstandard interactions between electrons and positrons and the plasma could increase the effective mass of the electrons and positrons [27]. A higher effective inmedium electron mass would cause the precipitous decline in electron and positron numbers seen in Fig. 1 to occur at a higher temperature, in turn, resulting in the strong decrease of $\mathcal{R}_{e^{ \pm}}$seen in Fig. 2 occurring at a higher temperature as well. The result is a higher annihilation freeze-out temperature. The increase in the effective electron/positron mass due to finite temperature QED corrections is too small to significantly change the annihilation freeze-out temperature, so BSM physics would be required to produce such an effect. The effects of the higher freeze-out temperature on $N_{\text {eff }}$ and BBN yields will be the same as those discussed in the previous scenario, but the particular BSM models will also affect BBN yields and possibly $N_{\text {eff }}$ through altered nuclear physics or fundamental constants [32-34].

In conclusion, we have computed the collision integral for electron-positron annihilation throughout the weak decoupling and BBN epochs of the early universe. We find that electrons and positrons fall out of local chemical equilibrium with the photons at a sufficiently late time such that there will be no signature of this freeze-out on $N_{\text {eff }}$ or BBN yields. However, one should be cautioned that nonstandard cosmologies may result in nontrivial consequences of this annihilation freeze-out.

\section{ACKNOWLEDGMENTS}

We thank G. Fuller for useful discussions. C. K. and L. T. acknowledge support from NSF Grant No. PHY-1812383. E. G. acknowledges support from NSF Grant No. PHY1630782 and the Heising-Simons Foundation, Grant No. 2017-228.

\section{APPENDIX A: EQUATIONS OF MOTION}

Throughout this work we have assumed that the electron and positron distributions are described by a Fermi-Dirac spectrum, parametrized by a temperature, $T$, and degeneracy parameter, $\eta=\mu / T$,

$$
f(p ; T, \eta)=\frac{1}{e^{E_{p} / T-\eta}+1},
$$

with $E_{p}=\sqrt{p^{2}+m_{e}^{2}}$, and $T$ is the common temperature of all particles in the plasma. This should be an excellent assumption since electromagnetic scattering between the 
elements of the photon-electron-positron-baryon plasma occurs at a rate much greater than the Hubble rate throughout the calculation.

To self-consistently determine the equations of motion for $T(t)$ and $\eta(t)$, we need to calculate the energy density, $\rho$, pressure, $P$, and number density, $n$, of electrons and positrons from the distribution functions:

$$
\begin{gathered}
\rho(T, \eta)=\int_{0}^{\infty} \frac{p^{2} d p}{\pi^{2}} E_{p} f(p ; T, \eta), \\
P(T, \eta)=\int_{0}^{\infty} \frac{p^{2} d p}{\pi^{2}} \frac{p^{2}}{3 E_{p}} f(p ; T, \eta), \\
n(T, \eta)=\int_{0}^{\infty} \frac{p^{2} d p}{\pi^{2}} f(p ; T, \eta) .
\end{gathered}
$$

These quantities can be expressed in terms of dimensionless functions by defining $\epsilon=p / T$ and $x=m_{e} / T$,

$$
\begin{gathered}
I_{1}(x, \eta)=\frac{\rho}{T^{4}}=\frac{1}{\pi^{2}} \int_{0}^{\infty} d \epsilon \epsilon^{2} E_{\epsilon} f(\epsilon ; x, \eta), \\
I_{2}(x, \eta)=\frac{P}{T^{4}}=\frac{1}{3 \pi^{2}} \int_{0}^{\infty} d \epsilon \frac{\epsilon^{4}}{E_{\epsilon}} f(\epsilon ; x, \eta), \\
N(x, \eta)=\frac{n}{T^{3}}=\frac{1}{\pi^{2}} \int_{0}^{\infty} d \epsilon \epsilon^{2} f(\epsilon ; x, \eta),
\end{gathered}
$$

where $E_{\epsilon}=\sqrt{\epsilon^{2}+x^{2}}$. Further, define the partial derivatives of these functions

$$
\begin{array}{ll}
J_{i}(x, \eta)=-\frac{\partial I_{i}}{\partial x}, & K_{i}(x, \eta)=\frac{\partial I_{i}}{\partial \eta}, \\
L(x, \eta)=-\frac{\partial N}{\partial x}, & M(x, \eta)=\frac{\partial N}{\partial \eta},
\end{array}
$$

with $i=1,2$. Each of these functions are, by design, dimensionless and positive definite.

\section{Chemical equilibrium}

In the chemical equilibrium solution, $\eta_{e^{-}}=\eta$ and $\eta_{e^{+}}=-\eta$. The total entropy density is

$s=T^{3}\left(I_{1}^{-}+I_{2}^{-}+I_{1}^{+}+I_{2}^{+}+\frac{4 \pi^{2}}{45}-\eta N^{-}+\eta N^{+}\right)+s_{\nu}$.

In the preceding equation, the "-" superscript corresponds to using the electron degeneracy parameter as the appropriate argument for each function, e.g., $I_{1}^{-}=I_{1}(x, \eta)$, while the "+" superscript corresponds to using the positron degeneracy parameter, e.g., $I_{1}^{+}=I_{1}(x,-\eta)$. While the entropy in the neutrino seas changes through weak interactions beyond weak decoupling, it is a small effect that does not impact our results. We therefore assume that neutrinos are decoupled from the plasma, so that the total entropy in the neutrino seas in a comoving volume, $s_{\nu} a^{3}$, is separately conserved.

The conservation of entropy in a comoving volume produces the differential equation

$$
\begin{aligned}
& {\left[\left(I_{1}^{-}+I_{2}^{-}+I_{1}^{+}+I_{2}^{+}-\eta\left(N^{-}-N^{+}\right)+\frac{4 \pi^{2}}{45}\right) \frac{3}{T}+\left(J_{1}^{-}+J_{2}^{-}+J_{1}^{+}+J_{2}^{+}-\eta\left(L^{-}-L^{+}\right)\right) \frac{m_{e}}{T^{2}}\right] \frac{d T}{d t}} \\
& \quad+\left[K_{1}^{-}+K_{2}^{-}-K_{1}^{+}-K_{2}^{+}-\left(N^{-}-N^{+}\right)-\eta\left(M^{-}+M^{+}\right)\right] \frac{d \eta}{d t}+\left[I_{1}^{-}+I_{2}^{-}+I_{1}^{+}+I_{2}^{+}-\eta\left(N^{-}-N^{+}\right)+\frac{4 \pi^{2}}{45}\right] \frac{d a}{d t}=0 .
\end{aligned}
$$

The evolution of the quantity $\left(n_{e^{-}}-n_{e^{+}}\right) a^{3}$, Eq. (11), yields the differential equation:

$$
\begin{aligned}
& {\left[\left(N^{-}-N^{+}\right) \frac{3}{T}+\left(L^{-}-L^{+}\right) \frac{m_{e}}{T^{2}}\right] \frac{d T}{d t}+\left(M^{-}+M^{+}\right) \frac{d \eta}{d t}} \\
& \quad+\left(N^{-}-N^{+}\right) \frac{3}{a} \frac{d a}{d t}=\frac{n_{b} a^{3}}{T^{3} a^{3}} \frac{d Y_{e}}{d t} .
\end{aligned}
$$

The quantity, $n_{b} a^{3}$, is the number of baryons in a comoving volume that is constant and equal to its initial value.

The evolution of $Y_{e}$ due to weak interactions, Eqs. (6)-(8), is

$$
\begin{aligned}
\frac{d Y_{e}}{d t}= & \left(1-Y_{e}\right)\left(\lambda_{\nu_{e} n}+\lambda_{e^{+} n}+\lambda_{n}\right) \\
& -Y_{e}\left(\lambda_{e^{-} p}+\lambda_{\bar{\nu}_{e} p}+\lambda_{\bar{\nu}_{e} e^{-} p}\right) .
\end{aligned}
$$

Each of the weak rates is an integral over the electron distributions [25] and depends on $T$ and $\eta$.

The evolution of the scale factor is given by the Friedmann equation,

$$
\frac{1}{a} \frac{d a}{d t}=\sqrt{\frac{8 \pi}{3 m_{p l}^{2}}}\left[\left(I_{1}^{-}+I_{1}^{+}+\frac{\pi^{2}}{15}\right) T^{4}+\frac{7 \pi^{2}}{40} T_{\mathrm{cm}}^{4},\right]^{1 / 2},
$$


where $T_{\mathrm{cm}}=T_{i}\left(a_{i} / a\right)$, and we have neglected the baryon energy density.

These four coupled differential equations, Eqs. (A11)(A14) define the evolution of the four dependent variables, $T, \eta, Y_{e}$, and $a$ using the initial conditions discussed in Sec. II.

It should be noted, however, that $Y_{e}$ can be calculated directly (without a differential equation) from

$$
Y_{e}=\frac{T^{3} a^{3}\left(N^{-}-N^{+}\right)}{n_{b} a^{3}}
$$

We chose to treat $Y_{e}$ as a dynamical variable with its own differential equation, but confirm that the above relationship between $Y_{e}, T, a$, and $\eta$ holds.

\section{Out of chemical equilibrium}

When we no longer assume chemical equilibrium, we are left with two independent degeneracy parameters, $\eta_{e^{-}}=\eta^{-}$ and $\eta_{e^{+}}=\eta^{+}$. The total entropy density is

$s=T^{3}\left(I_{1}^{-}+I_{2}^{-}+I_{1}^{+}+I_{2}^{+}+\frac{4 \pi^{2}}{45}-\eta^{-} N^{-}-\eta^{+} N^{+}\right)+s_{\nu}$.

We use the same notation as previously used in this appendix, where - corresponds to electrons, e.g., $I_{1}^{-}=$ $I_{1}\left(x, \eta^{-}\right)$, and + corresponds to positrons, e.g., $I_{1}^{+}=$ $I_{1}\left(x, \eta^{+}\right)$. The conservation of entropy in a comoving volume yields

$$
\begin{aligned}
& {\left[\left(I_{1}^{-}+I_{2}^{-}+I_{1}^{+}+I_{2}^{+}-\eta^{-} N^{-}-\eta^{+} N^{+}+\frac{4 \pi^{2}}{45}\right) \frac{3}{T}+\left(J_{1}^{-}+J_{2}^{-}+J_{1}^{+}+J_{2}^{+}-\eta^{-} L^{-}-\eta^{+} L^{+}\right) \frac{m_{e}}{T^{2}}\right] \frac{d T}{d t}} \\
& \quad+\left(K_{1}^{-}+K_{2}^{-}-N^{-}-\eta^{-} M^{-}\right) \frac{d \eta^{-}}{d t}+\left(K_{1}^{+}+K_{2}^{+}-N^{+}-\eta^{+} M^{+}\right) \frac{d \eta^{+}}{d t} \\
& \quad+\left(I_{1}^{-}+I_{2}^{-}+I_{1}^{+}+I_{2}^{+}-\eta^{-} N^{-}-\eta^{+} N^{+}+\frac{4 \pi^{2}}{45}\right) \frac{d a}{d t}=0 .
\end{aligned}
$$

The most easily distinguished difference between this equation and the corresponding one in the equilibrium scenario, Eq. (A11), are the separate degeneracy parameters, $\eta^{-}$and $\eta^{+}$, and their derivatives.

Likewise, in this scenario, the evolution of $\left(n_{e^{-}}-n_{e^{+}}\right) a^{3}$ yields the differential equation:

$$
\left[\left(N^{-}-N^{+}\right) \frac{3}{T}+\left(L^{-}-L^{+}\right) \frac{m_{e}}{T^{2}}\right] \frac{d T}{d t}+M^{-} \frac{d \eta^{-}}{d t}-M^{+} \frac{d \eta^{+}}{d t}+\left(N^{-}-N^{+}\right) \frac{3}{a} \frac{d a}{d t}=\frac{n_{b} a^{3}}{T^{3} a^{3}} \frac{d Y_{e}}{d t} .
$$

The evolution of $Y_{e}$ and $a$, Eqs. (A13) and (A14), remain unchanged. However, it should be noted that the weak rates depend on both $\eta^{-}$and $\eta^{+}$.

Now that there are two independent degeneracy parameters, we require an extra independent differential equation. We introduced the evolution of $\left(n_{e^{+}}+n_{e^{-}}\right) a^{3}$, Eq. (22), which results in the differential equation:

$$
\begin{aligned}
& {\left[\left(N^{-}+N^{+}\right) \frac{3}{T}+\left(L^{-}+L^{+}\right) \frac{m_{e}}{T^{2}}\right] \frac{d T}{d t}+M^{-} \frac{d \eta^{-}}{d t}+M^{+} \frac{d \eta^{+}}{d t}+\left(N^{-}-N^{+}\right) \frac{3}{a} \frac{d a}{d t}} \\
& \quad=\frac{n_{b} a^{3}}{T^{3} a^{3}}\left[\left(1-Y_{e}\right)\left(\lambda_{\nu_{e} n}-\lambda_{e^{+} n}+\lambda_{n}\right)+Y_{e}\left(-\lambda_{e^{-} p}+\lambda_{\bar{\nu}_{e} p}-\lambda_{\bar{\nu}_{e} e^{-} p}\right)+2 \frac{\mathcal{N} a^{3}}{n_{b} a^{3}}\right] .
\end{aligned}
$$

As before, the $n_{b} a^{3}$ is left explicitly uncanceled in the expressions above because it is constant. The net electron-positron annihilation rate, $\mathcal{N}$, is defined in Eq. (25).

The five coupled differential equations, Eqs. (A13), (A14), (A17)-(A19), define the evolution of five dependent variables, $T, \eta^{-}, \eta^{+}, Y_{e}$, and $a$, whose initial conditions and solutions are discussed in Sec. IV.

\section{APPENDIX B: SIMPLIFICATION OF THE COLLISION INTEGRAL}

This appendix details the reduction of the collision integral for electron-positron annihilation from a nine-dimensional integral to three-dimensional in a manner similar to the appendix in Ref. [1]. For the annihilation process, $e^{-}+e^{+} \rightleftharpoons \gamma+\gamma$, the Klein-Nishina amplitude is [35]

$$
\left\langle|\mathcal{M}|^{2}\right\rangle=2 e^{4}\left[\frac{P \cdot K_{2}}{P \cdot K_{1}}+\frac{P \cdot K_{1}}{P \cdot K_{2}}+2 m_{e}^{2}\left(\frac{1}{P \cdot K_{1}}+\frac{1}{P \cdot K_{2}}\right)-m_{e}^{4}\left(\frac{1}{P \cdot K_{1}}+\frac{1}{P \cdot K_{2}}\right)^{2}\right] \equiv M,
$$


where $e$ is the elementary charge, $m_{e}$ is the electron mass, $P$ and $Q$ are the four-momenta of the electron and positron, respectively, and $K_{1}$ and $K_{2}$ are the photon fourmomenta. We define the four-momenta of the electron as $P=(E, \mathbf{p})$ with particle energy $E$ and three-momentum p such that $P \cdot P=E^{2}-p^{2}=m_{e}^{2}$, with $p=|\mathbf{p}|$, and the same convention for positrons. Likewise, for the photon, $K=(k, \mathbf{k})$ with photon three-momentum $\mathbf{k}$ and energy $k=|\mathbf{k}|$.

The collision integral is

$$
\begin{aligned}
C= & \frac{1}{2 E_{p}} \int \frac{d^{3} q}{(2 \pi)^{3} 2 E_{q}} \frac{d^{3} k_{1}}{(2 \pi)^{3} 2 k_{1}} \frac{d^{3} k_{2}}{(2 \pi)^{3} 2 k_{2}} \\
& \times(2 \pi)^{4} \delta^{(4)}\left(P+Q-K_{1}-K_{2}\right) M\left(P \cdot K_{1}, P \cdot K_{2}\right) \\
& \times F\left(E_{p}, E_{q}, k_{1}, k_{2}\right),
\end{aligned}
$$

and

$$
\begin{aligned}
F\left(E_{p}, E_{q}, k_{1}, k_{2}\right) & \equiv F_{c}-F_{a} \\
= & f_{\gamma}\left(k_{1}\right) f_{\gamma}\left(k_{2}\right)\left[1-f_{e^{-}}\left(E_{p}\right)\right]\left[1-f_{e^{+}}\left(E_{q}\right)\right] \\
& -f_{e^{-}}\left(E_{p}\right) f_{e^{+}}\left(E_{q}\right)\left[1+f_{\gamma}\left(k_{1}\right)\right]\left[1+f_{\gamma}\left(k_{2}\right)\right]
\end{aligned}
$$

is the statistical factor for the creation $\left(F_{c}\right)$ and annihilation $\left(F_{a}\right)$ of electrons and positrons with the appropriate Fermi blocking and Bose enhancement terms. For the case $F=F_{a}$, we define $C_{a}$ as the collision integral solely for the annihilation pathway $\left(e^{-} e^{+} \rightarrow \gamma \gamma\right)$ and $F=F_{c}$ defines $C_{c}$ for the creation pathway $\left(\gamma \gamma \rightarrow e^{-} e^{+}\right)$.

Integrating over $\mathbf{k}_{2}$ reduces the delta function to a onedimensional energy-conserving delta function:

$$
\begin{aligned}
C= & \frac{1}{(2 \pi)^{5} 16 E_{p}} \int \frac{d^{3} q}{E_{q}} \int \frac{d^{3} k_{1}}{k_{1} k_{2}} \delta\left(E_{p}+E_{q}-k_{1}-k_{2}\right) \\
& \times\left. M\left(P \cdot K_{1}, P \cdot K_{2}\right) F\left(E_{p}, E_{q}, k_{1}, k_{2}\right)\right|_{k_{2}=\left|\mathbf{p}+\mathbf{q}-\mathbf{k}_{1}\right|},
\end{aligned}
$$

where $K_{2}=\left(k_{2}, \mathbf{p}+\mathbf{q}-\mathbf{k}_{1}\right)$, and $k_{2}$ is no longer an independent variable of integration, but is related to the other integration variables through momentum conservation, and

$$
k_{2}^{2}=|\mathbf{p}+\mathbf{q}|^{2}+k_{1}^{2}-2|\mathbf{p}+\mathbf{q}| k_{1} \cos \theta_{1},
$$

defining the new integration angle $\theta_{1}$ angle between the vectors $\mathbf{k}_{1}$ and $\mathbf{p}+\mathbf{q}$.

We need to evaluate the four-dot products

$$
P \cdot K_{1}=E_{p} k_{1}-\mathbf{p} \cdot \mathbf{k}_{1},
$$

$$
\begin{aligned}
P \cdot K_{2} & =E_{p} k_{2}-\mathbf{p} \cdot\left(\mathbf{p}+\mathbf{q}-\mathbf{k}_{1}\right) \\
& =E_{p} k_{2}-p^{2}-\mathbf{p} \cdot \mathbf{q}+\mathbf{p} \cdot \mathbf{k}_{1},
\end{aligned}
$$

which requires us to parametrize the dot products $\mathbf{p} \cdot \mathbf{q}$ and $\mathbf{p} \cdot \mathbf{k}_{1}$ in terms of the variables of integration. First, define $\theta_{q}$ as the angle between $\mathbf{p}$ and $\mathbf{q}$ so that

$$
\begin{gathered}
\mathbf{p} \cdot \mathbf{q}=p q \cos \theta_{q} \\
|\mathbf{p}+\mathbf{q}|=\left(p^{2}+q^{2}+2 p q \cos \theta_{q}\right)^{1 / 2} .
\end{gathered}
$$

The vector $\mathbf{p}+\mathbf{q}$ is in the plane generally defined by $\mathbf{p}$ and $\mathbf{q}$, but $\mathbf{k}_{1}$ is not necessarily in this plane. This means $\mathbf{k}_{1}$ is defined by both $\theta_{1}$, the angle between it and $\mathbf{p}+\mathbf{q}$, and an azimuthal angle $\phi_{1}$ measured out of this plane. To determine $\mathbf{p} \cdot \mathbf{k}_{1}$, we need to introduce another angle, $\psi$, between $\mathbf{p}$ and $\mathbf{p}+\mathbf{q}$. It follows that

$\mathbf{p} \cdot \mathbf{k}_{1}=p k_{1}\left(\cos \psi \cos \theta_{1}-\sin \psi \sin \theta_{1} \cos \phi_{1}\right)$,

with $\quad \cos \psi=\frac{\mathbf{p} \cdot(\mathbf{p}+\mathbf{q})}{p|\mathbf{p}+\mathbf{q}|}=\frac{p+q \cos \theta_{q}}{\sqrt{p^{2}+q^{2}+2 p q \cos \theta_{q}}}$.

This parametrizes the amplitude in terms of almost all the integration variables:

$$
M\left(P \cdot K_{1}, P \cdot K_{2}\right)=M\left(p, q, \theta_{q}, k_{1}, \theta_{1}, \phi_{1}\right) .
$$

When considering $d^{3} q=q^{2} d q d \cos \theta_{q} d \phi_{q}$ and $d^{3} k=$ $k^{2} d k d \cos \theta_{1} d \phi_{1}$, the integrand is independent of $\phi_{q}$, so this integral can be performed, which results in a factor of $2 \pi$, so

$$
\begin{aligned}
C= & \frac{1}{(2 \pi)^{4} 16 E_{p}} \int \frac{q^{2} d q d \cos \theta_{q}}{E_{q}} \int \frac{k_{1}^{2} d k_{1} d \cos \theta_{1} d \phi_{1}}{k_{1} k_{2}} \\
& \times \delta\left(E_{p}+E_{q}-k_{1}-k_{2}\right) M\left(p, q, \theta_{q}, k_{1}, \theta_{1}, \phi_{1}\right) \\
& \times F\left(E_{p}, E_{q}, k_{1}, k_{2}\right) .
\end{aligned}
$$

To complete the integral over $\theta_{1}$, we introduce the $u$ substitution,

$$
u^{2}=k_{2}^{2} \Rightarrow 2 k_{2} d u=-2|\mathbf{p}+\mathbf{q}| k_{1} d \cos \theta_{1} .
$$

This allows us to rewrite the collision integral as

$$
\begin{aligned}
C= & \frac{1}{(2 \pi)^{4} 16 E_{p}} \int \frac{q^{2} d q d \cos _{q}}{E_{q}|\mathbf{p}+\mathbf{q}|} \int d k_{1} d \phi_{1} \\
& \times \int_{a}^{b} d u \delta\left(E_{p}+E_{q}-k_{1}-u\right) M\left(p, q, \theta_{q}, k_{1}, \theta_{1}, \phi_{1}\right) \\
& \times F\left(E_{p}, E_{q}, k_{1}, u\right),
\end{aligned}
$$


with

$$
\begin{aligned}
& a=\left[|\mathbf{p}+\mathbf{q}|^{2}+k_{1}^{2}-2|\mathbf{p}+\mathbf{q}| k_{1}\right]^{1 / 2}=|| \mathbf{p}+\mathbf{q}\left|-k_{1}\right|, \\
& b=\left[|\mathbf{p}+\mathbf{q}|^{2}+k_{1}^{2}+2|\mathbf{p}+\mathbf{q}| k_{1}\right]^{1 / 2}=|\mathbf{p}+\mathbf{q}|+k_{1} .
\end{aligned}
$$

The delta function is nonzero on the interval $[a, b]$ only if

$$
a<E_{p}+E_{q}-k_{1}<b
$$

which will constrain the range of the other integrals once the delta function is resolved. To determine these ranges we need to consider two cases:

Case 1: $k_{1}<|\mathbf{p}+\mathbf{q}|$. The inequality has two parts: first,

$$
\begin{gathered}
|\mathbf{p}+\mathbf{q}|-k_{1}<E_{p}+E_{q}-k_{1} \\
\Rightarrow|\mathbf{p}+\mathbf{q}|<E_{p}+E_{q} .
\end{gathered}
$$

This is always true because $|\mathbf{p}+\mathbf{q}|<p+q$ (triangle inequality), and momenta are always less than their corresponding energy. Further, the second inequality

$$
\begin{aligned}
& E_{p}+E_{q}-k_{1}<|\mathbf{p}+\mathbf{q}|+k_{1} \\
& \quad \Rightarrow k_{1}>\frac{1}{2}\left(E_{p}+E_{q}-|\mathbf{p}+\mathbf{q}|\right) \equiv k_{\min } .
\end{aligned}
$$

The right-hand side is always positive, as stated previously. This reduces the range of the $k_{1}$ integral such that its integrand is nonzero.

Case 2: $k_{1}>|\mathbf{p}+\mathbf{q}|$. This case differs from Case 1 only in the first inequality,

$$
\begin{aligned}
& k_{1}-|\mathbf{p}+\mathbf{q}|<E_{p}+E_{q}-k_{1} \\
& \quad \Rightarrow k_{1}<\frac{1}{2}\left(E_{p}+E_{q}+|\mathbf{p}+\mathbf{q}|\right) \equiv k_{\max },
\end{aligned}
$$

and again, the second inequality yields $k_{1}>k_{\min }$.

Putting the cases together, we find that the delta function is nonzero when $k_{\min }<k_{1}<k_{\max }$, so upon evaluating the $d u$ integral the delta function fixes $\cos \left(\theta_{1}\right)$ in terms of other variables:

$$
\begin{aligned}
\cos \theta_{1} & =\frac{|\mathbf{p}+\mathbf{q}|^{2}+k_{1}^{2}-k_{2}^{2}}{2 k_{1}|\mathbf{p}+\mathbf{q}|} \\
& =\frac{|\mathbf{p}+\mathbf{q}|^{2}-\left(E_{p}+E_{q}\right)^{2}+2 k_{1}\left(E_{p}+E_{q}\right)}{2 k_{1}|\mathbf{p}+\mathbf{q}|} .
\end{aligned}
$$

The collision integral then becomes

$$
\begin{aligned}
C= & \frac{1}{(2 \pi)^{4} 16 E_{p}} \int \frac{q^{2} d q d \cos _{q}}{E_{q}|\mathbf{p}+\mathbf{q}|} \int_{k_{\min }}^{k_{\max }} d k_{1} \\
& \times\left[\int_{0}^{2 \pi} d \phi_{1} M\left(p, q, \theta_{q}, k_{1}, \theta_{1}, \phi_{1}\right)\right] F\left(E_{p}, E_{q}, k_{1}, k_{2}\right),
\end{aligned}
$$

where, once again, $k_{2}=E_{p}+E_{q}-k_{1}$. Note that only $M$ depends on $\phi_{1}$, so we can perform the integral in the square brackets analytically. To simplify our notation, we define the function

$$
\tilde{M}\left(p, q, \theta_{q}, k_{1}\right)=\frac{1}{(2 \pi)\left(2 e^{4}\right)} \int_{0}^{2 \pi} d \phi_{1} M\left(p, q, \theta_{q}, k_{1}, \theta_{1}, \phi_{1}\right) .
$$

The amplitude, Eq. (B1), depends only on the four-dot products $P \cdot K_{1}$ and $P \cdot K_{2}$. These dot products can be written in the form $A+B \cos \phi_{1}$. The function $\tilde{M}$, above, can be analytically integrated as long as $A>B$. These fourdot products can be shown to be positive definite, which proves that $A>B$, by considering the annihilation process in the center-of-momentum frame where the four-momenta can be written as $P=\left(E_{p}, \mathbf{p}\right), Q=\left(E_{p},-\mathbf{p}\right), K_{1}=(k, \mathbf{k})$, and $K_{2}=(k,-\mathbf{k})$,

$$
\begin{aligned}
& P \cdot K_{1}=k\left(E_{p}+p \cos \vartheta\right)>0, \\
& P \cdot K_{2}=k\left(E_{p}-p \cos \vartheta\right)>0,
\end{aligned}
$$

where $\vartheta$ is the angle between $\mathbf{p}$ and $\mathbf{k}$. This means that the individual terms in the amplitude are positive definite in any frame and allow us to analytically perform the integral resulting in

$$
\begin{gathered}
\tilde{M}\left(p, q, \theta_{q}, k_{1}\right)=-2+\left(3 m_{e}^{2}+E_{p} E_{q}-p q \cos \theta_{q}\right)\left(\frac{1}{\sqrt{X^{2}-Y^{2}}}+\frac{1}{\sqrt{Z^{2}-Y^{2}}}\right) \\
-m_{e}^{4}\left[\frac{X}{\left(X^{2}-Y^{2}\right)^{3 / 2}}+\frac{Z}{\left(Z^{2}-Y^{2}\right)^{3 / 2}}+\frac{2}{X+Z}\left(\frac{1}{\sqrt{X^{2}-Y^{2}}}+\frac{1}{\sqrt{Z^{2}-Y^{2}}}\right)\right], \\
X=E_{p} k_{1}-p k_{1} \cos \psi \cos \theta_{1},
\end{gathered}
$$




$$
\begin{gathered}
Y=p k_{1} \sin \psi \sin \theta_{1}, \\
Z=m_{e}^{2}+E_{p} E_{q}-p q \cos \theta_{q}-X,
\end{gathered}
$$

$\psi$ and $\theta_{1}$ are defined in terms of $p, q, \theta_{q}$, and $k_{1}$ in Eqs. (B11) and (B22).

The final form of the collision integral is therefore

$$
C=\frac{e^{4}}{(2 \pi)^{3} 8 E_{p}} \int_{0}^{\infty} \frac{q^{2} d q}{E_{q}} \int_{-1}^{1} \frac{d \cos \theta_{q}}{|\mathbf{p}+\mathbf{q}|} \int_{k_{\min }}^{k_{\max }} d k_{1} \tilde{M}\left(p, q, \theta_{q}, k_{1}\right) F\left(E_{p}, E_{q}, k_{1}, E_{p}+E_{q}-k_{1}\right) .
$$

This integral is calculated using Gauss-Legendre quadrature for the two inner integrals $\left(\cos \theta_{q}, k_{1}\right)$ over finite ranges and using Gauss-Laguerre quadrature for the outer integral $(q)$ over the infinite range.

[1] E. Grohs, G. M. Fuller, C. T. Kishimoto, M. W. Paris, and A. Vlasenko, Phys. Rev. D 93, 083522 (2016).

[2] P. F. de Salas and S. Pastor, J. Cosmol. Astropart. Phys. 07 (2016) 051.

[3] G. Mangano, G. Miele, S. Pastor, T. Pinto, and P.D. S. Ofelia Pisanti, Nucl. Phys. B729, 221 (2005).

[4] A. D. Dolgov, S. H. Hansen, S. Pastor, S. T. Petcov, G. G. Raffelt, and D. V. Semikoz, Nucl. Phys. B632, 363 (2002).

[5] K. N. Abazajian et al. (CMB-S4 Collaboration), arXiv: 1610.02743.

[6] R. Maiolino et al., arXiv:1310.3163.

[7] E. Grohs, G. M. Fuller, C. T. Kishimoto, and M. W. Paris, J. Cosmol. Astropart. Phys. 05 (2015) 017.

[8] X. Chu, B. Dasgupta, M. Dentler, J. Kopp, and N. Saviano, J. Cosmol. Astropart. Phys. 11 (2018) 049.

[9] A. Fradette, M. Pospelov, J. Pradler, and A. Ritz, Phys. Rev. D 90, 035022 (2014).

[10] J. Berger, K. Jedamzik, and D. G. E. Walker, J. Cosmol. Astropart. Phys. 11 (2016) 032.

[11] S. D. McDermott, Phys. Rev. Lett. 120, 221806 (2018).

[12] G. M. Fuller, C. T. Kishimoto, and A. Kusenko, arXiv:1110 .6479 .

[13] K. Jedamzik, Phys. Rev. D 74, 103509 (2006).

[14] K. Jedamzik and M. Pospelov, New J. Phys. 11, 105028 (2009).

[15] M. Kawasaki, K. Kohri, and T. Moroi, Phys. Rev. D 71, 083502 (2005).

[16] M. Kawasaki, K. Kohri, T. Moroi, and Y. Takaesu, Phys. Lett. B 751, 246 (2015).

[17] M. Kawasaki, K. Kohri, T. Moroi, and Y. Takaesu, Phys. Rev. D 97, 023502 (2018).

[18] E. W. Kolb and M. S. Turner, The Early Universe (AddisonWesley, Reading, MA, 1990).
[19] J. Birrell, C. T. Yang, and J. Rafelski, Nucl. Phys. B890, 481 (2015).

[20] A. D. Dolgov, S. H. Hansen, and D. V. Semikoz, Nucl. Phys. B503, 426 (1997).

[21] G. Mangano, G. Miele, S. Pastor, and M. Peloso, Phys. Lett. B 534, 8 (2002).

[22] J. J. Bennett, G. Buldgen, M. Drewes, and Y. Y. Y. Wong, arXiv:1911.04504.

[23] E. Grohs, G. M. Fuller, C. T. Kishimoto, and M. W. Paris, Phys. Rev. D 95, 063503 (2017).

[24] L. Kawano, NASA STI/Recon, Tech. Rep. 92-25163, 1992.

[25] E. Grohs and G. M. Fuller, Nucl. Phys. B911, 955 (2016).

[26] P. A. R. Ade et al. (Planck Collaboration), Astron. Astrophys. 594, A13 (2016).

[27] E. Grohs and G. M. Fuller, Nucl. Phys. B923, 222 (2017).

[28] A. Arbey, J. Auffinger, K. P. Hickerson, and E. S. Jenssen, arXiv:1806.11095.

[29] R. Consiglio, P. F. de Salas, G. Mangano, G. Miele, S. Pastor, and O. Pisanti, Comput. Phys. Commun. 233, 237 (2018).

[30] C. Pitrou, A. Coc, J.-P. Uzan, and E. Vangioni, Phys. Rep. 04, 005 (2018).

[31] J. Froustey and C. Pitrou, arXiv:1912.09378.

[32] C. M. Müller, G. Schäfer, and C. Wetterich, Phys. Rev. D 70, 083504 (2004).

[33] T. Dent, S. Stern, and C. Wetterich, Phys. Rev. D 76, 063513 (2007).

[34] M. E. Mosquera and O. Civitarese, Phys. Rev. C 96, 045802 (2017).

[35] M.E. Peskin and D. V. Schroeder, An Introduction to Quantum Field Theory (Westview Press, Boulder, CO, 1995). 